\title{
The Effects of Social Structure, Geographical Structure, and Population Size on the Evolution of Mitochondrial DNA: II. Molecular Clocks and the Lineage Sorting Period
}

\author{
Guy A. Hoelzer, ${ }^{1-3}$ Joel Wallman, ${ }^{4}$ Don J. Melnick ${ }^{5,6}$ \\ ${ }^{1}$ Department of Biology University of Nevada Reno, Reno, NV 89557, USA \\ ${ }^{2}$ Department of Environmental and Resource Sciences, University of Nevada Reno, Reno, NV 89557, USA \\ ${ }^{3}$ Graduate Program in Ecology, Evolution and Conservation Biology, University of Nevada Reno, \\ Reno, NV 89557, USA \\ ${ }^{4}$ The Harry Frank Guggenheim Foundation, New York, NY, USA \\ ${ }^{5}$ Department of Anthropology, Columbia University, New York, NY 10027, USA \\ ${ }^{6}$ Center for Environmental Research and Conservation, Columbia University, New York, NY 10027, USA
}

Re: J Mol Evol (1998) 47:21-31. There is some confusion in Hoelzer et al. (1998) about the relationship between the length of the lineage sorting period (LSP) calculated from their simulations and the fixation time (FT) treated elsewhere in the literature. Conceptually, the LSP and FT are identical. Tajima (1990) used a very different kind of simulation to show that the average FT for nuclear mutations is $4 \mathrm{~N}$ under neutral conditions. Applying the logic of Tajima's model, the FT for mtDNA alleles would be $2 \mathrm{~N}_{f}$, on average, not $4 \mathrm{~N}_{f}$ as reported by Hoelzer et al.; where $\mathrm{N}_{f}$ is the number of females in the population. Yet Hoelzer et al. also report that, under similar neutral conditions, their simulations found LSPs close to $4 \mathrm{~N}$. The source of this difference appears to lie in the differing mechanisms used in the two simulations to effect changes in allele (haplotype) frequencies across generations. Tajima used the telescoping method of Kimura and Takahata (1983), employing a diffusion model of allele frequency change in which the variance of possible frequencies in the next generation is tied to the initial allele frequency. In contrast, the simulation model of Hoelzer et al. tracked the survival and reproductive fate of each individual (Wallman et al. 1996).
Each adult female gave birth to two daughters, which were then subjected to the survival probabilities associated with the mother's dominance rank and assigned by the computer operator. Assigned survival probabilities were scaled to preserve a dynamic equilibrium in overall population size. The method used by Hoelzer et al. is an idiosyncratic way of implementing stochastic variance in survival among individuals within a class that results in a lower potential for allele frequency change between generations than the telescoping method implemented by Tajima. Hence, it is not surprising that the average "null" LSPs observed by Hoelzer et al. are longer than the $2 \mathrm{~N}_{f}$ that would be predicted by Tajima's model. Which of the two methods provides a more realistic distribution of allele frequency change in particular systems is an empirical question. The observations of model behavior and the relationships among parameters reported by Hoelzer et al. should generalize to other theoretical treatments without any surprising influence of the unusual mechanism used to create stochastic lineage expansion and contraction. I am grateful to Matt Hare for pointing out the discrepancy between Tajima's results and ours in such a clear manner as to facilitate this understanding. 


\section{References}

Hoelzer GA, Wallman J, Melnick DJ (1998) The effects of social structure, geographical structure, and population size on the evolution of mitochondrial DNA: II. molecular clocks and the lineage sorting period. J Mol Evol 47:21-31

Kimura M, Takahata N (1983) Selective constraint in protein polymorphism: study of the effectively neutral mutation model by using an improved pseudosampling method. Proc Natl Acad Sci USA 80:1048-1052

Tajima F (1990) Relationship between DNA polymorphism and fixation time. Genetics 125:447-454

Wallman J, Hoelzer GA, Melnick DJ (1996) The effects of social structure, geographical structure, and population size on the evolution of mitochondrial DNA: a simulation model. CABIOS 12:481-489 